\title{
Collisional Rayleigh-Taylor instability and shear-flow in equatorial Spread-F plasma
}

\author{
N. Chakrabarti ${ }^{1}$ and G. S. Lakhina ${ }^{2}$ \\ ${ }^{1}$ Saha Institute of Nuclear Physics, 1/AF, Bidhannagar, Kolkata- 700 064, India \\ ${ }^{2}$ Indian Institute of Geomagnetism, Dr. Nannabhai Moos Marg, Colaba, Mumbai - 400 05, India
}

Received: 18 April 2002 - Revised: 7 November 2002 - Accepted: 14 November 2002

\begin{abstract}
Collisional Rayleigh-Taylor (RT) instability is considered in the bottom side of the equtorial F-region. By a novel nonmodal calculation it is shown that for an applied shear flow in equilibrium, the growth of the instability is considerably reduced. Finite but small amounts of diffusion enhances the stabilization process. The results may be relevant to the observations of long-lived irregularities at the bottomside of the F-layer.
\end{abstract}

Key words. Ionosphere (ionospheric irregularities, equatorial ionosphere, plasma waves and instabilities)

\section{Introduction}

The Rayleigh-Taylor (RT) instability has been extensively studied in a wide range of physical context both experimentally and theoretically (Amatucci et al., 1996; Aron et al., 1976). The density fluctuations in the nighttime equatorial F-region of the ionosphere, commonly referred to as Equatorial Spread-F (ESF), have been a topic of active research in the last few decades. Apart from the academic point of view, there is a practical interest due to the fact that satellite communication and navigation systems are seriously disrupted by the rapid fluctuations of the signal amplitude that is induced by the electron density fluctuations associated with ESF (Basu, 1997). It is well known that when the altitude of the F-layer is high enough and the density gradient is steep, the large-scale density fluctuations grow on the bottom side via density gradient driven instability when the gravity is directed against the density gradient. This instability is commonly known as electrostatic interchange or RT instability and invoked to explain large-scale density fluctuations (Flaherty et al., 1999).

The primary source by which this instability is triggered is gravitational force acting on an inverted density gradient (e.g.

Correspondence to: G. S. Lakhina

(lakhina@iig.iigm.res.in) a heavy fluid supported by a light fluid). The basic mechanism of this instability, an interchange of flux tube to tap the gravitational free energy, is the same mechanism that drives Rayleigh-Benard instability in the thermal convection of a gravitationally unstable fluid. In this case the mean temperature gradient of the fluid plays a similar role as the density gradient and the buoyancy force acts similar to the gravity.

Analytical studies of the RT mode with shear-flow has been studied previously. Normally such analysis is done by a standard "modal approach". In this traditional method perturbed quantities are expanded in Fourier integrals. The modes are assumed to have an exponential time behavior and if the imaginary part of the frequency is positive, the modes will grow in time, resulting in an instability. This method is well known and effective to solve problems involving the Hermitian operator, which gives an orthogonal eigenfunction. But the operator with shear-flow is generally non-Hermitian and the eigenfunctions are not orthogonal to each other. A detailed discussion is recently given by Tatsuno et al. (2001). Therefore, using the common Fourier or Laplace method in a system with shear flow, we may overlook the secular and transient behavior of the mode which can lead to an incorrect result even in the linear regime. In this paper, we use the "non-modal" approach to solve collisional RT mode in the presence of transverse shear flow. The method, originally proposed by Lord Kelvin, became popular recently and is extensively used in a variety of problems (Hassam, 1992). In this method, one considers the temporal evolution of spatial Fourier harmonics of the perturbations without any spectral expansion in time. The wave number of each spatial Fourier harmonics along the flow shear varies in time. This method is useful to analyze various physical phenomena involving shear flow which describe the energy exchange between shear flow and the perturbations.

The influence of transverse velocity shear on RayleighTaylor instability has been well investigated in the linear theory by various authors (Guzdar et al., 1982; Satyanarayana et al., 1984, 1987; Chaturvedi and Ossakow, 1977). It is found that a sheared velocity flow can substantially reduce 
the growth rate of a Rayleigh-Taylor instability in the short wavelength regime. They have also discussed the application of this result in ionospheric plasma (e.g. equatorial Spread-F and ionospheric plasma clouds). It is also known that the RT mode may self-consistently generate a velocity shear which can then stabilize the mode (Finn et al., 1992; Chakrabarti and Lakhina, 2001).

Rayleigh-Taylor instabilities, as they are applied to the ionosphere, can be divided into two categories: collisional and inertial. In the collisional limit the ion-neutral frequency is dominant, and $v_{\text {in }} \gg \omega$, where $v_{\text {in }}$ is the ion-neutral collision frequency and $\omega$ is the mode frequency; in the inertial limit $\omega \gg v_{\text {in }}$ and the neutral collision may be neglected. However, we restrict our discussion in this study to the collisional domain only. In this paper we study the effect of externally applied velocity shear on the Rayleigh-Taylor mode, to gain a better understanding on the theories of RT mode dynamics. We discuss the linear collisional RT instability by nonmodal calculation (or Kelvin's methods of shearing modes), to demonstrate the effect of shear-flow on this mode. Our calculation demonstrates that equilibrium shear-flow can considerably reduce the growth of the instability. But if one adds a small diffusion term, the mode can be stabilized completely. This is a new aspect in the nonmodal solution that has not been studied before.

The rest of the paper is organized as follows. In Sect. 2 a simple physical model for collisional RT instability is presented and the basic equations are derived. We have discussed the self-consistent equilibrium profile of density and velocity and then re-derived the dispersion relation for the RT mode in the collisional regime. In Sect. 3 the effect of shear-flow is presented analytically. We conclude in Sect. 4 with a discussion of our results.

\section{Model, basic equations and equilibrium}

Development of a total self-consistent and comprehensive theory which describes the nonlinear properties of the RT mode applied to the ionosphere is very difficult in many aspects. In this report we have taken the vastly used and best illustrated model existing in the literature (Hassam et al., 1986; Keskinen et al., 1979).

The nonlinear fluid equations used to describe the electrostatic RT instability are the continuity and momentum equations for the electrons and ions. We model these equations in a three-dimensional slab geometry with the ambient magnetic field directed in the $z$ direction $\left(\boldsymbol{B}=\hat{e}_{z} B_{0}\right)$. For the purpose of relating this geometry to the ionospheric situations we employ the standard convention where $x$ is vertically upward (in the direction of the ambient density gradient, $N_{0}=N_{0}(x), N_{0}^{-1} d N_{0} / d x=L_{n}^{-1}>0$, where $L_{n}$ is the density gradient scale length) and $y$ is eastward, which will be referred to as the horizontal or zonal direction. The gravitational acceleration is in the $-x$ direction $\left(g=-g \hat{e}_{x}\right)$. For this situation the plasma is unstable to RT instability, since $\boldsymbol{g} \cdot \nabla N_{0}<0$.
For the system described above, the flute type $(\partial / \partial z=0$, no variation along the magnetic field), RT instability is expected to be the fastest growing instability. The fluid equations can be simplified by the following assumptions. We assume that electrons are warm and ions are cold. The assumption of cold ions, i.e. the neglect of the ion temperature, is a shortcoming, because this eliminates the ion diamagnetic drift which introduces the finite Larmor radius effect (FLR) that could play a stabilizing role in RT instability, particularly in the short wavelength regime $k \rho_{i} \gg 1$, (where $\rho_{i}$ is the mean ion Larmor radius and $k$ is a typical wave number). One advantage of setting $T_{i} \ll T_{e}$ is that the kinetic effect, such as Landau damping will play no role and the system is well described by the fluid model. The wave electric field is virtually electrostatic, since the plasma pressure is much smaller than the ambient magnetic energy density. The electron and ion densities are equal (quasi-neutrality condition), since we are interested in wavelengths much larger than the Debye length. We assume a background neutral density to exist with $v_{i n}$, the ion-neutral collision frequency, and the electron-neutral collision frequency is neglected. Since in the F-region $v_{e n} / \Omega_{e} \ll v_{i n} / \Omega_{i}\left(\Omega_{j}\right.$ is the cyclotron frequency of the species $j$ ), we assume $v_{i n} \ll \Omega_{i}$. The electron and ion inertial effect is neglected since we are only interested in collisional regime $\left(\omega \ll v_{i n}\right)$. With these restrictions, the RT instability in the F-region of the ionosphere can be described by the following set of equations (Huba et al., 1985):

$$
\begin{aligned}
& \frac{\partial N}{\partial t}+\nabla \cdot\left(N \boldsymbol{V}_{j}\right)=0 \\
& 0=-\frac{e}{m_{e}}\left(\boldsymbol{E}+\frac{1}{c} \boldsymbol{V}_{e} \times \boldsymbol{B}\right)-\frac{T_{e}}{m_{e}} \frac{\nabla N}{N}-v_{e i}\left(\boldsymbol{V}_{e}-\boldsymbol{V}_{i}\right) \\
& 0=\frac{e}{m_{i}}\left(\boldsymbol{E}+\frac{1}{c} \boldsymbol{V}_{i} \times \boldsymbol{B}\right)+\boldsymbol{g}-v_{i n} \boldsymbol{V}_{i}-v_{i e}\left(\boldsymbol{V}_{i}-\boldsymbol{V}_{e}\right) \\
& \nabla \cdot \boldsymbol{J} \equiv \nabla \cdot\left[N e\left(\boldsymbol{V}_{i}-\boldsymbol{V}_{e}\right)\right]=0
\end{aligned}
$$

where the various symbols have their usual meaning (Huba et al., 1985; Chakrabarti and Lakhina, 2001). Solving electron momentum Eq. (2) for their directed velocities, we find to the lowest order (Sudan and Keskinen, 1984)

$$
\boldsymbol{V}_{e}=\frac{c}{B_{0}} \hat{e}_{z} \times \nabla \varphi-\frac{c T_{e}}{e B_{0}} \hat{e}_{z} \times \nabla \ln N-\frac{\nu_{e i}}{\Omega_{e}} \frac{c_{s}^{2}}{\Omega_{i}} \nabla\left(\frac{\tilde{n}}{N_{0}}\right),(5)
$$

which consists of $\boldsymbol{E} \times \boldsymbol{B}$ and the diamagnetic drift velocity. The last term arises due to the frictional force between electrons and ions, $T_{e}$ is the electron temperature, $\varphi(\boldsymbol{E}=-\nabla \varphi)$ is the electrostatic potential, $\tilde{n}$ is the density fluctuations over equilibrium $N_{0}$. Note here that we have taken $v_{e i}$ as a small parameter during the perturbative solution of the momentum equation. $v_{e i}$ is only effective during perturbation, and its role in the equilibrium is unimportant. Similarly, the ion velocity is determined from Eq. (3) and is given by

$$
\boldsymbol{V}_{i}=\frac{g}{\Omega_{i}} \hat{e}_{y}+\frac{c}{B_{0}} \hat{e}_{z} \times \nabla \varphi-\frac{c \nu_{i n}}{B_{0} \Omega_{i}} \nabla_{\perp} \varphi-\frac{\nu_{e i}}{\Omega_{e}} \frac{c_{s}^{2}}{\Omega_{i}} \nabla\left(\frac{\tilde{n}}{N_{0}}\right),
$$


where the terms on the right-hand side are gravitational drift, $\boldsymbol{E} \times \boldsymbol{B}$ drift, Pedersen drift and drift due to electrons and ions friction, respectively. In solving $\boldsymbol{V}_{i}$, we have neglected the ion viscous terms $\left(\sim v_{i i}\right)$, since they are smaller than the Pedersen drift contribution $\left(\sim v_{i n}\right)$. Here, $c_{s}^{2}=T_{e} / m_{i}$ is the ion sound speed and $\tilde{n}$ is the fluctuating part of the density over the equilibrium $N_{0}$. By substitution of these velocities into Eqs. (4) and (1) we arrive at the coupled set of equations for the potential $(\varphi)$ and density $(N)$ :

$$
\begin{aligned}
& \frac{c}{B_{0}}\left[\nabla_{\perp}^{2} \varphi+\nabla_{\perp} \varphi \cdot \nabla_{\perp} \ln N\right]=\frac{g}{v_{i n}} \frac{\partial}{\partial y} \ln N \\
& \left(\frac{\partial}{\partial t}+\frac{c}{B_{0}} \hat{e}_{z} \times \nabla \varphi \cdot \nabla_{\perp}\right) \ln N=D_{\perp} \nabla_{\perp}^{2}\left(\frac{\tilde{n}}{N_{0}}\right) .
\end{aligned}
$$

Note that in the density Eq. (8), $D_{\perp}=v_{e i} \rho_{e}^{2}$ arises because electron ion collision introduces an effective damping rate and we assume it is negligible in equilibrium. In the above equations $\rho_{e}=v_{t e} / \Omega_{e}, v_{t e}=\sqrt{T_{e} / m_{e}}$ and $\Omega_{e, i}=\left(e B_{0} / m_{e, i} c\right)$.

Now we shall discuss ambient density and velocity profiles. In the absence of equilibrium flow, variation in density as a function of $x$ may be taken arbitrarily and that satisfies the basic equations. Since in equilibrium both $N_{0}(x)$ and $\varphi_{0}(x)$ are functions of $x$ only, we have the equilibrium equation as follows from Eqs. (7) and (8) as

$$
\begin{aligned}
& \frac{d}{d x}\left[N_{0}(x) \frac{c}{B_{0}} \frac{d \varphi_{0}}{d x}\right]=0, \\
& \frac{\partial \varphi_{0}}{\partial x} \frac{\partial N_{0}}{\partial y}-\frac{\partial \varphi_{0}}{\partial y} \frac{\partial N_{0}}{\partial x}=0 .
\end{aligned}
$$

Note that Eq. (10) automatically satisfies for $N_{0}$, with $\varphi_{0}$ being any function of $x$. From Eq. (9) we have

$N_{0}(x) V_{0}(x)=n_{0} v_{0}$,

where $V_{0}(x)=\left(c / B_{0}\right)\left(d \varphi_{0} / d x\right)$ is the equilibrium velocity and $n_{0}, v_{0}$ are constants. Let us assume that

$N_{0}(x)=n_{0}\left(1-\frac{x}{L_{n}}\right)^{-1}$

with $x / L_{n} \ll 1$ and $L_{n}$ equilibrium density scale length. With this $N_{0}(x)$ we find the normalized $V(x)=v_{0}-v_{0}^{\prime} x$, where $v_{0}=\left(c \varphi_{0}^{\prime} / B_{0}\right)\left(v_{i n} L_{n} / g L_{\perp}\right), v_{0}^{\prime}=v_{0} / L_{n}$ are constants and $L_{\perp}$ is the characteristic scale length in the perpendicular direction.

Next we write the basic equations for the perturbations in dimensionless form. For this purpose the normalizations are used as follows:

$$
\begin{aligned}
& t\left(\frac{g}{v_{i n} L_{n}}\right) \rightarrow t ; \frac{x, y}{L_{\perp}} \rightarrow x, y ; \frac{\tilde{n}}{N_{0}}\left(\frac{L_{n}}{L_{\perp}}\right) \rightarrow n ; \\
& \frac{\varphi}{B_{0} L_{\perp}}\left(\frac{c v_{i n}}{g}\right)\left(\frac{L_{n}}{L_{\perp}}\right) \rightarrow \phi ; \frac{D_{\perp}}{L_{\perp}^{2}}\left(\frac{L_{n} v_{i n}}{g}\right) \rightarrow D .
\end{aligned}
$$

With these normalized variables Eqs. (7) and (8) may be rewritten as

$$
\begin{aligned}
& \nabla_{\perp}^{2} \phi=\frac{\partial n}{\partial y}, \\
&\left(\frac{\partial}{\partial t}-v_{0}^{\prime}\left(x-x_{0}\right) \frac{\partial}{\partial y}+\hat{e}_{z} \times \nabla \phi \cdot \nabla_{\perp}\right) n-\frac{\partial \phi}{\partial y} \\
& \quad=D \nabla_{\perp}^{2} n .
\end{aligned}
$$

In deriving Eqs. (11) and (12) we have used $\ln N \approx$ $\ln N_{0}(x)+\tilde{n} / N_{0}$ and $\left(\ln N_{0}\right)^{\prime}=1 / L_{n}>0$ and also used the ordering $\rho_{s} \ll L_{\perp} \ll L_{n}$, where $\rho_{s}$ is the ion Larmor radius at electron temperature $\left[\rho_{s}=\left(\sqrt{T_{e} / m_{i}}\right) / \Omega_{i}\right]$. Due to the above mentioned ordering, Eq. (11) becomes simple since we neglect the terms proportional to $L_{\perp} / L_{n}$. Note also that in the equilibrium, shear convective flow has a null at $x=x_{0}\left(=v_{0} / v_{0}^{\prime}\right)$.

\subsection{Linear instability}

Here we shall recapitulate the local $\left(k_{x} L_{\perp} \gg 1\right)$ linear dispersion of the collisional RT mode. The linear dispersion equation of the system is obtained from the linear version of Eqs. (11) and (12) and assuming that the perturbed quantities vary as $\exp \left(i k_{x} x+i k_{y} y-i \omega t\right)$, where $k_{x}$ and $k_{y}$ are positive integers. The well-known (Hassam et al., 1986) dispersion equation is given by

$\omega=i\left(\frac{k_{y}^{2}}{k_{\perp}^{2}}-D k_{\perp}^{2}\right)$,

where $k_{\perp}^{2}=k_{x}^{2}+k_{y}^{2}$. The boundary between linearly stable and unstable regions of $\boldsymbol{k}$ space is given by $D=k_{y}^{2} / k_{\perp}^{4}$. The maximum growth rate is obtained for $k_{x}=0$.

In the absence of any dissipation we find the usual maximum growth rate as $\gamma=g /\left(v_{i n} L_{n}\right)$ (Guzdar et al., 1982; Hassam et al., 1986). In the next section we would like to study the nonmodal analysis of shear-flow effect on this instability.

\section{Shear-flow effect on Collisional RT instability}

In this section we demonstrate by a simple analytic calculation of how the collisional RT instability is suppressed by velocity shear. The basic nonlinear equations for this analysis with the equilibrium flow as mentioned before may be written as

$$
\nabla_{\perp}^{2} \phi=\frac{\partial n}{\partial y},
$$

$$
\begin{aligned}
& \left(\frac{\partial}{\partial t}-v_{0}^{\prime}\left(x-x_{0}\right) \frac{\partial}{\partial y}+\hat{e}_{z} \times \nabla \phi \cdot \nabla_{\perp}\right) n-\frac{\partial \phi}{\partial y} \\
& \quad=D \nabla_{\perp}^{2} n .
\end{aligned}
$$




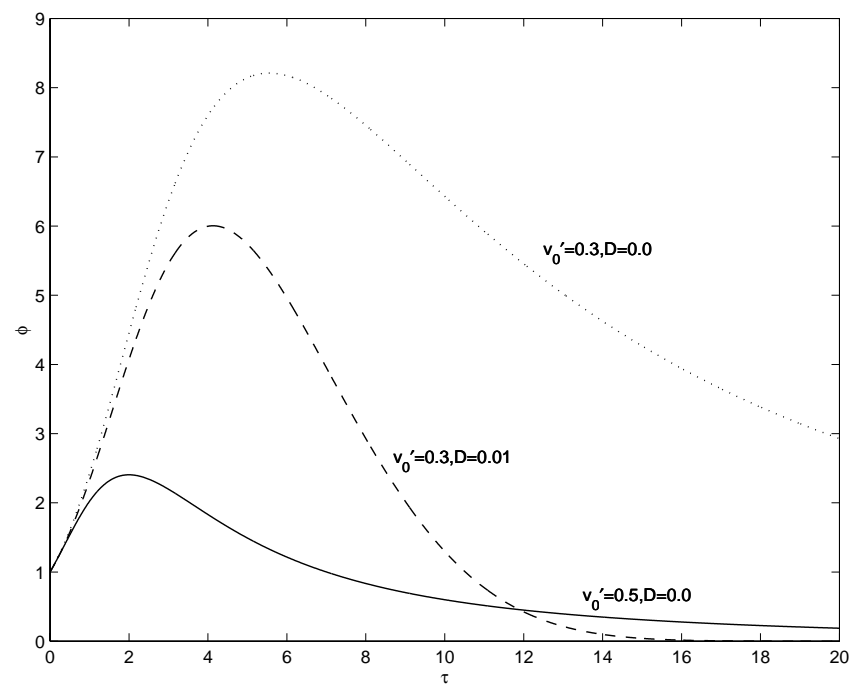

Fig. 1. Solution of Eq. (21) for normalized potential with and without dissipation is plotted for different velocity shear parameters for $k=2.0$. The different curves indicate the reduction of the growth of the RT mode by velocity shear with and without the presence of dissipation.

To solve the set of Eqs. (14) and (15) we use a spatiallyinhomogeneous Galilean transformation. Specifically, we introduce a spatio-temporal coordinate transformation to the fixed reference frame $x-y$ to obtain the local moving frame (Lagrangian) going with mean shear flow (Hassam, 1992),

$\xi=y-v_{0}^{\prime}\left(x-x_{0}\right) t$ and $\tau=t$.

Equation (16) induces the following transformations of the partial derivatives:

$\partial_{x}=-v_{0}^{\prime} \tau \partial_{\xi}, \quad \partial_{y}=\partial_{\xi}, \quad \partial_{t}=\partial_{\tau}-v_{0}^{\prime}\left(x-x_{0}\right) \partial_{\xi}$.

With the help of these transformations and assuming solutions of the form $\phi=\phi(\xi, \tau), n=n(\xi, \tau)$, we obtain

$\left(1+v_{0}^{\prime 2} \tau^{2}\right) \frac{\partial^{2} \phi}{\partial \xi^{2}}=-\frac{\partial n}{\partial \xi}$,

$\frac{\partial n}{\partial \tau}=-\frac{\partial \phi}{\partial \xi}+D\left(1+v_{0}^{\prime 2} \tau^{2}\right) \frac{\partial^{2} n}{\partial \xi^{2}}$.

Note that with the transformation used above the explicit $x$ dependences from the equations are eliminated, and as a result, the convective nonlinear terms vanish. This is expected since by this choice of coordinates we are reducing the dimensionality of the perturbations. Transformation of the partial derivatives shows that the shear flow term, together with $\partial_{t}$ reduces into a simple "temporal" derivative $\partial_{\tau}$. The set of Eqs. (17), (18) is homogeneous with respect to $\xi$. Therefore, a Fourier transform of $n, \phi$ with respect to $\xi$ yields a good quantum number $k$, and the equations may be solved by assuming the solution of the form $\exp (i k \xi)$. With this assumption the system may be simplified to a first order ordinary differential equation in $\tau$. This equation may be solved analytically to shear-flow effect on collisional RT mode. The equation for $\phi$ is given by

$\frac{d}{d \tau}\left(k_{\perp}^{2} \phi\right)=k^{2} \phi-D k_{\perp}^{4} \phi$,

where $k_{\perp}^{2}(t)=k^{2}\left(1+v_{0}^{\prime 2} \tau^{2}\right)$. Here the notation $k_{\perp}$ implies the perpendicular wave vector and it is time dependent. Note that this time dependence enters through finite velocity shear. The dissipative terms in the above equations can be eliminated with further transformations $\phi=$ $\left(\psi / k_{\perp}^{2}\right) \exp \left(-D \int k_{\perp}^{2}(\tau) d \tau\right)$. The simple differential equation for $\psi$ may then be written as:

$\frac{d \psi}{d \tau}-\frac{1}{1+v_{0}^{\prime 2} \tau^{2}} \psi=0$.

For $v_{0}^{\prime}=0$, if we assume the temporal mode behavior as $\psi \sim \exp (-i \omega t)$, then from Eq. (20) we recover the local dispersion relation for the collisional RT wave. In the absence of velocity shear $\left(v_{0}^{\prime}=0\right)$, the above equation can be solved easily for $\psi$ and recalling the transformations we find $\phi=\bar{\phi}_{0} \exp \left[g \tau /\left(v_{i n} L_{n}\right)\right] \exp \left(i k y-v_{e i} \rho_{e}^{2} k^{2} \tau\right)$ which clearly shows that potential fluctuations grow in time unless the damping rate overpowers the growth rate of the mode. Now our purpose is to show that this exponential growth is arrested by the finite velocity shear $\left(v_{0}^{\prime}\right)$.

Now writing the solution of Eq. (19) in the presence of $v_{0}^{\prime}$, we have

$\phi=$

$\frac{\bar{\phi}_{0}}{1+v_{0}^{\prime 2} \tau^{2}} \exp \left[\frac{1}{v_{0}^{\prime}} \tan ^{-1} v_{0}^{\prime} \tau-D k^{2} \tau\left(1+v_{0}^{\prime 2} \tau^{2} / 3\right)\right]$.

This implies that the velocity shear can reduce the exponential growth of the instability.

Therefore, it is clear that even for $D=0$, but in the presence of a finite shear flow, the solution exhibits stabilization of the collisional RT instability. This is shown below by taking the asymptotic limit $\left(v_{0}^{\prime} \tau \gg 1\right)$ of the Eq. (21)

$\phi \approx \frac{\bar{\phi}_{0}}{v_{0}^{\prime 2} \tau^{2}} \exp \left[\frac{1}{v_{0}^{\prime}}\left(\frac{\pi}{2}-\frac{1}{v_{0}^{\prime} \tau}\right)\right]$.

It is easy to see from the above equation that for large time $\tau \rightarrow \infty, \phi \rightarrow 0$, which implies a stabilization of the Collisional RT mode by equilibrium shear-flow. A small but finite amount of dissipation enhances the stabilization process, as is demonstrated in Fig. 1, which depicts the solution of Eq. (21) in the presence of velocity shear and dissipation.

\section{Discussions}

In this work we have shown that over a long time scale, the Rayleigh Taylor instability is considerably suppressed by shear flow in the equilibrium state. We have shown that for a small amount of diffusion the most unstable long wavelength modes are completely stabilized for an imposed velocity shear $v_{0}^{\prime}$. 
These results may be applicable to the long-lived irregularities which appear to be confined to the bottomside of the F-layer (Flaherty et al., 1999). Radar observations indicate a significant shear in the horizontal flow on the bottomside of the equatorial F-region (Kudeki et al., 1981; Kelley et al., 1986). It is plausible to put forward a scenario where the bottomside irregularities grow due to RT instability soon after sunset. In the absence of shear flow, these irregularities can attain large amplitudes as time passes, such that nonlinear terms dominate the dynamics, the bubble-type features develop, and the irregularities rise to higher heights. However, in the presence of shear flow, the initial exponential growth of the RT instability is arrested at a later time by the shear flow (see Fig. 1), so that the instability could not develop into bubbles. Therefore, these irregularities remain confined at the bottomside of the F-layer.

It should be emphasized that the calculation presented in this paper does not consider the flow-fluctuation theory, i.e. due to the nonlinear effect of how a flow profile changes and reacts back on the fluctuations self-consistently is not incorporated. As it is mentioned in the Introduction, our main objective in this paper to show, that for a given equilibrium velocity profile, how it affects the linear RT mode. It is important to mention that if one includes velocity curvature $\left(v_{0}^{\prime \prime}\right)$ in the equilibrium flow then the Kelvin Helmholtz mode is likely to be unstable. For a more realistic case one should also consider the influence of plasma dynamics along the geomagnetic field, which is considerably more significant than velocity curvature. Since a motion parallel to the magnetic field has a further stabilizing effect on the RT instability, one should include this for completeness. However, this calculation shows, for the most unstable RT instability, how velocity shear influences the growth rate of the mode. To carry out the self-consistent shear flow effect in a collisional rayleighTaylor mode in the presence of parallel plasma dynamics, a more deeper investigation is needed which we hope to report in the future.

Acknowledgements. Topical Editor M. Lester thanks M. Keskinen and another referee for their help in evaluating this paper.

\section{References}

Amatucci, W. E., Walker, D. N., Ganguli, G., et al.: Plasma response to strongly sheared flow, Phys. Rev. Lett., 77, 1978, 1996.

Arons, J. and Lea, S. M.: Accretion on to magnetized neutron stars: structure and interchange instability of a model magnetosphere,
Astrophys. J., 207, 914, 1976

Basu, B.: Generalized Rayleigh-Taylor instability in the presence of time dependent equilibrium, J. Geophys. Res., 102, 17305 , 1997.

Chakrabarti, N. and Lakhina, G. S.: Nonlinear saturation of Rayleigh-taylor instability and generation of shear flow in equatiorial Spread-F plasma, Nonl. Proc. Geophys., 8, 181-190, 2001.

Chaturvedi, P. K. and Ossakow, S. L.: Non-linear Theory of the Collisional Rayleigh-Taylor instability in Equatorial Spread-F, Geophys. res. Lett., 4, 558, 1977.

Finn, J. M., Drake, J. F., and Guzdar, P. N.: Instability of fluid vortices and generation of shear flow, Phys. Fluids B., 4, 2758, 1992.

Flaherty, J. P., Seyler, C. E., and Trefethen, L. N.: Large amplitude transient growth in the linear evolution of equatorial Spread-F with a sheared zonal flow, J. Geophys. Res., 104, 6843, 1999.

Guzdar, P. N., Satyanarayana, P., Huba, J. D., and Ossakow, S. L.: Influence of velocity shear on rayleigh-Taylor instability , Geophys. Res. Letts., 9, 547, 1982.

Hassam, A. B., Hall, W., Huba, J. D., and Keskinen, M. J.: Spectral Characteristics of Interchange Turbulence, J. Geophys. Res., 91, 13 513, 1986.

Hassam, A. B.: Nonlinear stabilization of the Rayleigh-Taylor instability by external velocity shear, Physics Fluids, 4, 485, 1992.

Huba, J. D., Hassam, A. B., Schwartz, I. B., and Keskinen, M. J.: Ionospheric turbulence: Interchange instabilities and chaotic fluid behavior, Geophys. Res. Lett. ,12, 65, 1985.

Kelly, M. C., LaBelle, T., Kudeki, E., Fejer, B. G., Basu, S., Basu, Su., Baker, K. D., Hanuise, C., Argo, P., Woodman, R. F., Swartz, W. E., Farley, D. T., and Meriwether, Jr., W.: The Condor equatorial Spread-F campaign: Overview and results of the large-scale measurements, J. Geophys. Res., 91, 5487, 1986.

Keskinen, M. J., Sudan, R. N., and Ferch, R. L.: Temporal and spatial power spectrum studies of numerical simulations of type II gradient drift irregularities in the equatorial electrojet, J. Geophys. Res., 84, 1419, 1979.

Kudeki, E, Fejer, B. J., Farley, D. T., and Ierkic, H. M.: Interferometer studies of equatorial F-region irregularities and drifts, Geophys. Res. Lett., 8, 377, 1981.

Satyanarayana, P., Guzdar, P. N., Huba, J. D., and Ossakow, S. L.: Rayleigh-Taylor instability in the presence of a stratified shear layer, J. Geophys. Res., 89, 2945, 1984.

Satyanarayana, P., Lee, Y. C., and Huba, J. D.: The stability of a stratified shear layer, Phys. Fluids., 30, 81, 1987.

Sudan, R. N. and Keskinen M. J.: Unified theory of the Power spectram of Interchange wavelength Ionospheric Electron density fluctuations, J. Geophys. Res., 89, 9840, 1984.

Tatsuno, T., Volponi, F., and Yoshida, Z.: Transient Phenomena and secularity of linear interchange instabilities with shear flow in homogeneous magnetic field plasmas, Phys. Plasmas, 8, 399, 2001 